\title{
Transient marine electromagnetic responses of 3D resistive structures: Implications for navigation
}

\author{
Andrei Swidinsky ${ }^{1}$ and R. Nigel Edwards ${ }^{2}$
}

\begin{abstract}
The marine controlled-source electromagnetic method has become a popular technique for mapping the electrical resistivity structure of the seafloor. Electromagnetic fields, produced by an electric dipole transmitter, diffuse through the earth and are recorded on the seabed by nodal or cable based electric dipole receivers. Accurate information on transmitter and receiver geometry is extremely important for proper interpretation; errors in the position and orientation of the transmitter and/or the receivers propagate into errors in the predicted seafloor resistivity. We consider the special situation where a receiver cable is towed in the vicinity of a transmitter which is located on the seafloor with a known position and orientation. We theoretically and numerically examine the response of this system for a selection of 1D, 2D, and 3D models, and show that although the electromagnetic field recorded at early times contains information about the subsurface resistivity, the late time decay of the transient response is only a function of the seawater conductivity and receiver position for all the models we consider. In fact, the shape of the late time decay is almost exactly the same as that of a dipole in a wholespace having the same resistivity as seawater. This shows that late time transient information can be used to determine the position of the receiver cable, whereas early time information can be used to determine the resistivity structure of the seafloor.
\end{abstract}

\section{INTRODUCTION}

A typical transient marine controlled-source electromagnetic (CSEM) system consists of an electric dipole transmitter and one or more electric dipole receivers. The objective of a CSEM survey is to determine the seafloor resistivity by recording the electromagnetic fields which diffuse through the earth from the transmitter to the receivers. Accurate information concerning the system geometry is crucial for proper interpretation; errors in the position and orientation of the transmitter and/or receivers propagate into errors in the predicted seafloor resistivity. At sea, establishing the position of instruments can be extremely challenging, time-consuming, and costly. However, several studies have shown that the measured electromagnetic fields themselves may be used to locate CSEM instruments on the seafloor. For example, Weitemeyer (2008) determines the position and orientation of a towed transmitter and nodal receivers using frequency domain electromagnetic measurements, and Key and Lockwood (2010) use an orthogonal Procrustes rotation to determine the orientation of nodal receivers on the seafloor. In Swidinsky and Edwards (2011), we show for certain multireceiver set-ups and 1D crustal electrical profiles that the system geometry and the seafloor resistivity may be determined independently. More specifically, we show that the transient electromagnetic fields produced by a transmitter with a known position and orientation can be used to establish the position of a cabled streamer of receivers towed by a ship or an autonomous underwater vehicle around the source (see Figure 1 for illustration). Such a permanent transmitter has been deployed by the University of Toronto to study gas hydrate deposits off the West Coast of Canada (Mir, 2011). The problem posed in Swidinsky and Edwards (2011) differs in two ways from the previous two studies. First, it considers transient electromagnetic measurements using a towed receiver cable, and second, it considers the special case in which the transmitter position is exactly known. Under these conditions, we conclude that the cable position and seafloor resistivity can be determined independently using electric field measurements.

This interesting conclusion has motivated us to attempt to understand the physics behind our observations. In its simplest form, our theory is that the late time arrival of the electromagnetic field corresponds to field diffusion through the seawater, and that this late time behavior should, in almost all cases, be virtually insensitive to the seafloor resistivity structure. Thus, a change in the late time arrival of the field should correspond to a change in the receiver

Manuscript received by the Editor 14 March 2012; revised manuscript received 30 July 2012; published online 10 December 2012.

${ }^{1}$ Helmholtz-Zentrum für Ozeanforschung Kiel (GEOMAR), Kiel, Germany. E-mail: aswidinsky@ geomar.de.

${ }^{2}$ University of Toronto, Department of Physics, Toronto, Canada. E-mail: edwards@core.physics.utoronto.ca.

(C) 2012 Society of Exploration Geophysicists. All rights reserved. 
cable position, but not the seafloor resistivity. The intent of the present paper is to test this basic theoretical model against results obtained from numerical calculations. We proceed by focussing on the following three questions: (1) What is the effect of seafloor structure on late time transient marine electromagnetic responses? (2) Do 2D or 3D structures affect these results? (3) What are the implications concerning instrument positioning using electromagnetic fields? We address these questions theoretically and numerically in the following three sections, using a selection of $1 \mathrm{D}, 2 \mathrm{D}$, and $3 \mathrm{D}$ earth models.

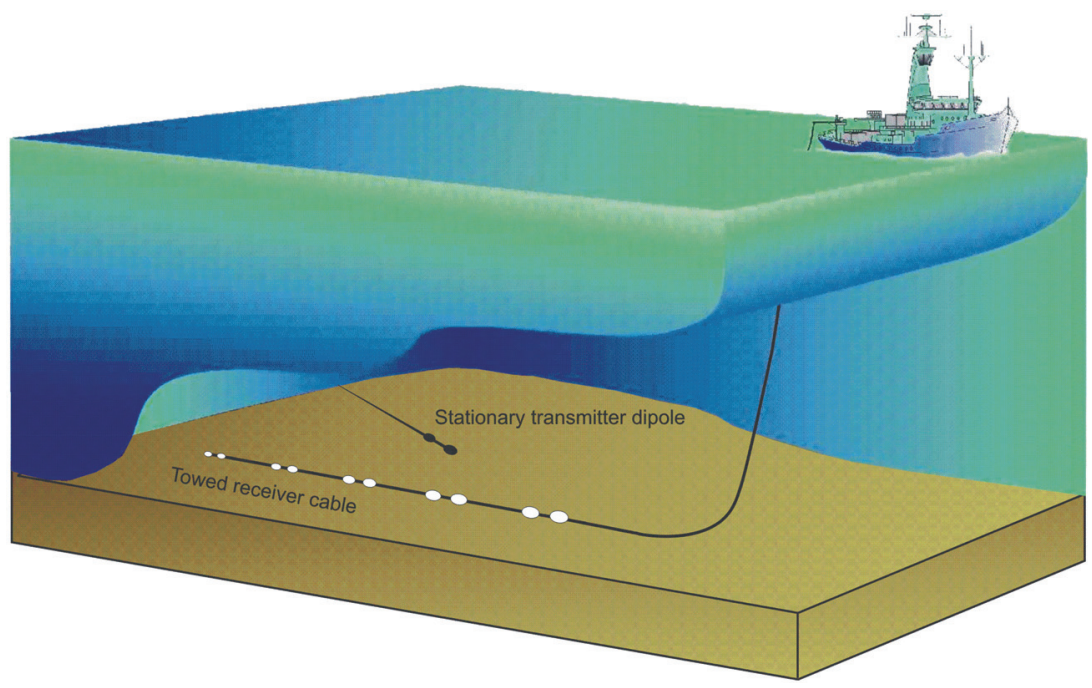

Figure 1. Illustration of the fixed-transmitter, towed-receiver, marine CSEM configuration.

\section{Early time}

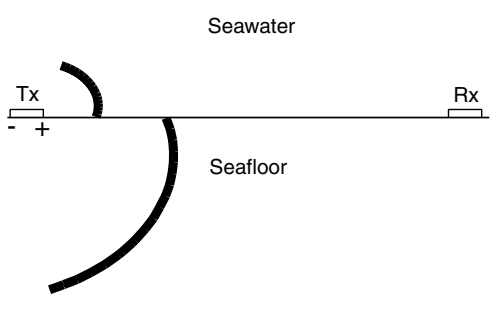

Late time

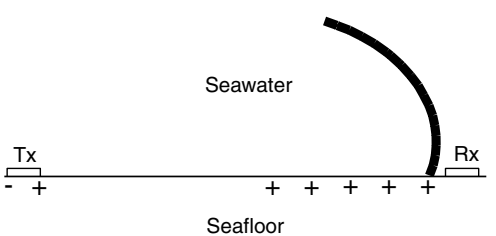

\section{Intermediate time}

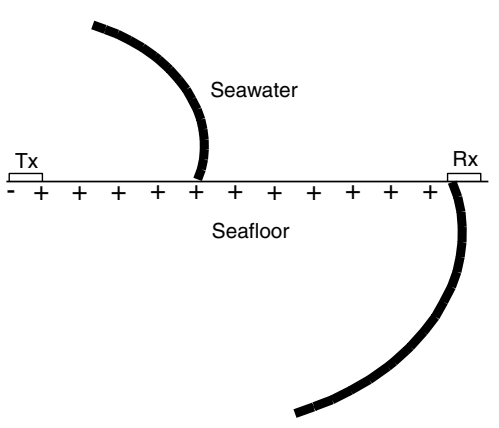

Static limit

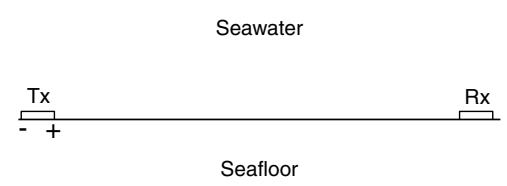

Figure 2. Inductive fields and charge distributions for a resistive seafloor half-space model at early, intermediate, and late times, as well as at the static limit. The region of induction is indicated by the energy front (perpendicular to the Poynting vector), whereas charge accumulation is indicated by + and - signs.

\section{THE PHYSICAL MODEL}

In general, the electric field recorded at a receiver on the seafloor is produced by two physical effects: induction and charge accumulation. Induction occurs throughout the earth, but only when the electromagnetic field varies with time. On the other hand, charge can only build up at interfaces separating regions of different conductivity and on the two poles of the transmitter. Unlike induction, charge accumulation can occur at all times, including the static limit.

In conductive media, induction produces eddy currents and corresponding electromagnetic fields which diffuse away from the transmitter at a rate inversely proportional to the conductivity of each region (i.e., the seawater, the seafloor sediments, as well as conductive or resistive zones within the seafloor). Therefore, in the common case of a resistive seafloor, induction will produce fields which move faster through the seafloor than through the relatively more conductive seawater. The receiver will record two transient events: The first corresponds to an arrival of the electromagnetic fields diffusing through the seafloor, and the second corresponds to a later arrival through the seawater. In the less common case of a conductive seafloor, the situation will be reversed; the first event will correspond to the fields which travel through the relatively less conductive seawater, and the second to the fields which travel through the much more conductive seafloor. In either case, these inductive fields will be recorded at a particular receiver position for a window of time before they diffuse away, and the inductive contribution to the total field is a transient effect which produces temporal variations at the receiver.

When the current is abruptly switched on in the transmitter, charge appears on the positive and negative electrodes of the bipole. As the electromagnetic fields produced by induction diffuse away from the source, they cause charge to also accumulate at interfaces between regions of different conductivity. As long as current is being transmitted, these charges and their corresponding fields will remain even after the inductive fields have diffused away. This static charge distribution produces the measured field at the DC limit. The charge contribution to the total field is therefore mainly a static effect which produces amplitude variations at the receiver.

The four time panels in Figure 2 illustrate the process described above for the case of a resistive seafloor half-space. In the early time panel, induction appears around the transmitter but it has spread further in the more resistive seafloor than in the conductive seawater. Charge has developed on the electrodes. In the intermediate time panel, the inductive fields have traveled through the seafloor and have reached the receiver, but they have only traveled a short distance 
through the seawater. The presence of the fields in the seafloor has caused charge to start accumulating at the interface. In the late time panel, the inductive fields in the seafloor have diffused away whereas the fields in the seawater have just reached the receiver. Charge has built up on the interface around the seawater energy front, and remains on the electrodes. Finally, in the static limit panel, the inductive fields have totally diffused away whereas the charge (and corresponding DC electric field) remains on the electrodes. The charge on the interface has disappeared due to the symmetry of this simple problem.

The field measured at the receiver at late time is the sum of the inductive response diffusing through the seawater and the charge accumulation on the electrodes and interfaces. The transient response occurring at late time, that is, the arrival time and subsequent decay of the inductive fields, is only a function of the seawater conductivity and transmitter-receiver offset. By contrast, the charge accumulation and corresponding fields, which are functions of the seawater conductivity and the seafloor structure, have no effect on the transient response; these elements only introduce a static shift to the recorded fields, much like the static shift observed in the magnetotelluric method (see, for example, Jones, 1988). Thus, in addressing our first question, we can argue that seafloor structure affects the late time response recorded at a receiver by introducing a static shift. However, these fields do not change the transient behavior of the fields at late time. By removing this static effect; for example, by taking the derivative of the step response, the seafloor-independent late time transient response should be easy to identify. Due to the similarity of this effect to magnetotelluric static shift, we hypothesize that the charge will affect the amplitude spectrum of the fields but not the phase characteristics, so that similar conclusions may apply in the frequency domain. However, this speculation has yet to be confirmed.

Moving on to more complex models, Figure 3 illustrates the same process, but for a 2D seafloor quarter-space. As in Figure 2, at early time, induction appears around the transmitter but has spread further in the seafloor than in the seawater. Charge has developed on the electrodes. At intermediate time, the inductive fields have traveled through the seafloor and reached the receiver, but these fields have been distorted as they pass the contact between quarter-spaces. They move faster in the more resistive quarterspace and slower in the more conductive adjacent region. Regardless of the inductive behavior in the seafloor, the transient field has only traveled a short distance through the seawater which has a higher conductivity than either quarter-space. Again, as in Figure 2, the fields in the seafloor have caused charge to start accumulating at the interfaces (on the seafloor and along the contact). Note that, along the contact, the charge is negative because the electric field is mainly directed from the resistor into the conductor. At late time, the inductive fields in the seafloor have diffused away whereas fields in the seawater have just reached the receiver, their time of arrival being determined by the seafloor conductivity, independent of seafloor structure. Charge has built up throughout the earth. At the static limit, the inductive fields have totally diffused away but the charge still remains, in this case on the electrodes and on the interfaces.

In Figure 4, we show a similar process for a complex 3D model which includes undulations in the seafloor. The same theoretical reasoning applies as in the previous two cases. At early time, there is local induction near the transmitter and charge accumulation on the bipole. At intermediate time, induction which occurs mainly in the seafloor, reaches the receiver but is distorted by the 3D geology. This causes further charge accumulation, including accumulation along the undulating seafloor and at the surface of the resistive target. At late time, just like in the previous two cases, induction occurs only in the seawater and charge accumulates throughout the entire area. Finally, at the static limit, there are only DC fields produced by the charge distribution.

Thus, in addressing our second question, we can argue that 2D and 3D structure will affect the charge distribution and induction within the seafloor, but will not alter the transient response caused by induction in the seawater at late times. The physics has not changed. We can proceed to argue that, because the transient response at late time is governed only by the transmitter-receiver offset and the seawater conductivity, a measure of the late time arrival and subsequent decay of the fields can be used to determine the position of an instrument on the seafloor. There may be pathological cases where these conclusions do not hold; for example, a situation where the seafloor is more conductive than the seawater, very shallow water, or certain transmitter-receiver geometries where induction through the seawater is minimized. In fact, we speculate that the first two cases will likely exhibit early time arrivals which are independent of the seafloor structure - a hypothesis that requires further examination. We are confident, however, that the physical model
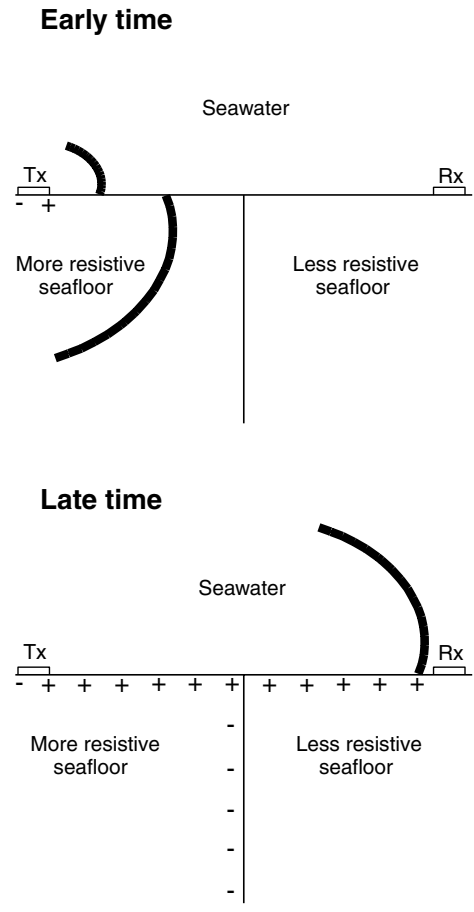

Figure 3. Inductive fields and charge distributions for a resistive seafloor quarter-space model. Note that the energy front moves faster in the more resistive quarter space and is distorted upon reaching the contact.

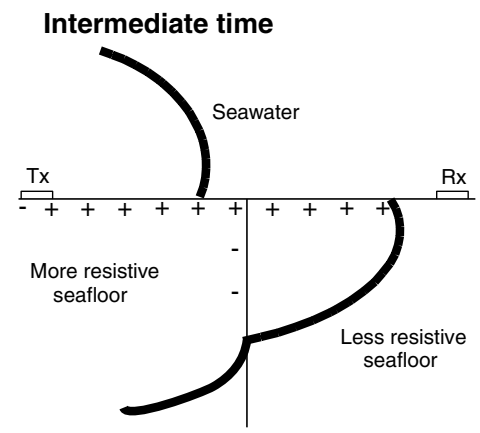

Static limit

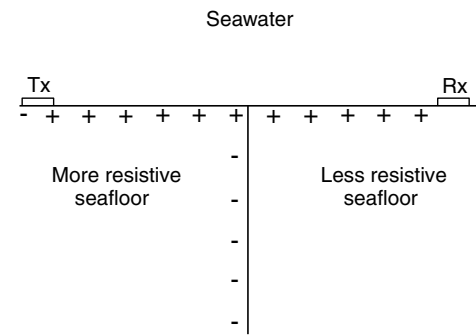




\section{Early time}

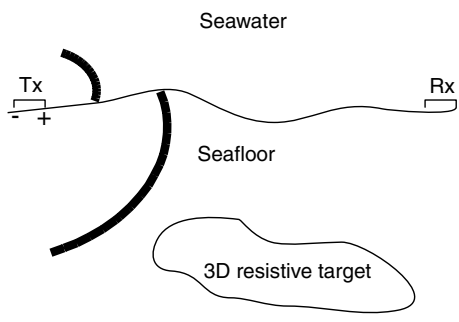

Late time
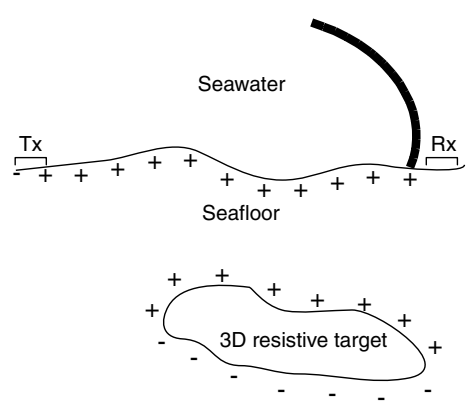

\section{Intermediate time}

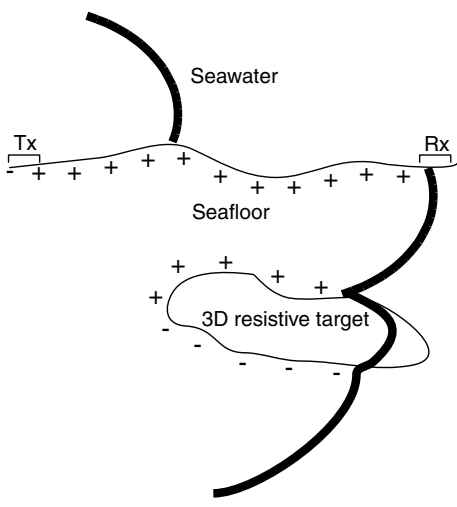

Static limit

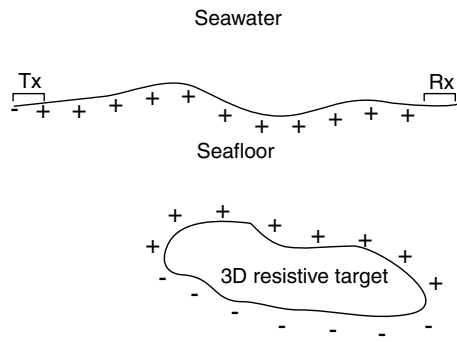

Figure 4. Inductive fields and charge distributions for a complex 3D model. Note that the induction in the seawater at late time, indicated by the energy front, is unaffected by features in the seafloor.

a)

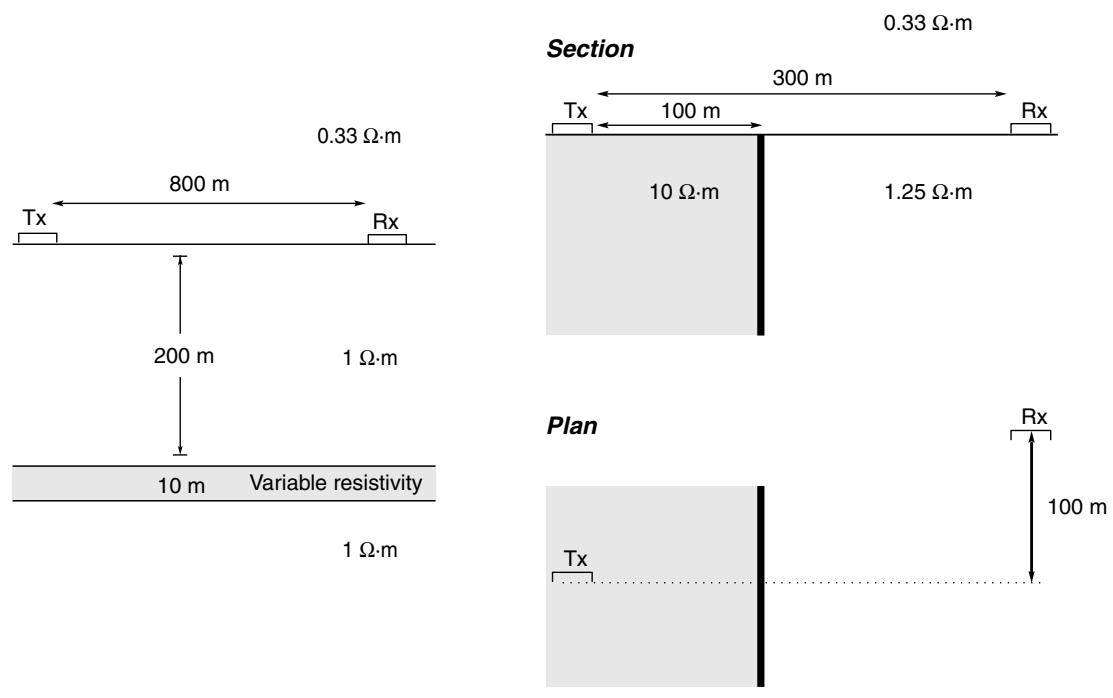

presented here describes a wide range of scenarios. Note that our arguments do not necessarily apply in the presence of induced polarization (IP) effects. We have assumed that a $100 \%$ duty cycle square wave produces the step-response. If a $50 \%$ duty cycle is used instead, the off-time could yield IP effects which may distort the late time transient behavior of our physical model. The topic of marine IP is beyond the scope of the present paper, but is discussed in detail in Davydycheva et al. (2006).

\section{A NUMERICAL MODEL STUDY}

Figure 5 shows three different seafloor models that we use to examine our theory. Figure 5a shows a simple uniform seafloor containing a layer of variable resistivity (model A), Figure 5b shows a 2D model of a seafloor quarter-space contact between two media of different resistivities (model B), and Figure 5c shows a 3D model of a resistive sheet buried within a uniform seafloor (model C). For each of these models, we calculate the log-differentiated step-response for different transmitter-receiver geometries. Because this type of response is similar to the impulse response of a system, we shall refer to it as the pseudoimpulse response (for further insight, see for example Edwards, 1997). Note that we choose different array geometries for the three different situations with the purpose of investigating if the conclusions of this study are not geometry dependent when the experiment is

c)
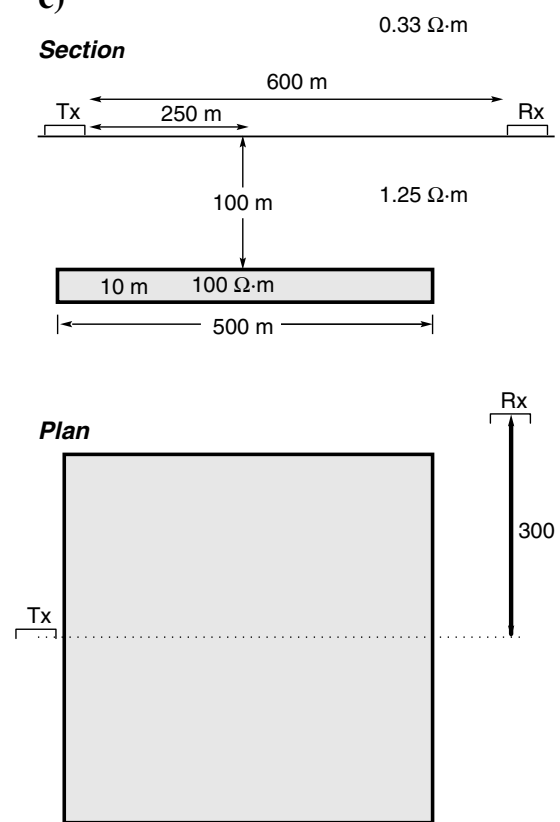

Figure 5. Three seafloor models used in this study. (a) A layer of variable resistivity buried in a uniform seafloor (model A). (b) A seafloor contact consisting of two quarter-spaces of different resistivity (model B). (c) A resistive sheet buried in a uniform seafloor (model C). 
appropriately designed. By "appropriately designed," we realize that some receiver tow paths or instrument positions may be unsuitable for location using electromagnetic fields, regardless of the structure of the seafloor; what we propose here is a navigational refinement procedure for roughly established cable positions with suitable source-receiver geometries.

Figure 6 shows the pseudoimpulse response for the three different models. For illustration purposes, in model A we consider the true electric field, whereas in models $\mathrm{B}$ and $\mathrm{C}$ the response has been normalized by the amplitude of the second arrival. The purpose of this normalization is to highlight the late time transient behavior of the pseudoimpulse response in marine settings, without the added complexity of interpreting amplitude information. The response of model A was calculated using conventional 1D modeling techniques (e.g., Edwards, 1997), the response of model B was calculated numerically using the finite-difference code SLDMEM3T (Druskin

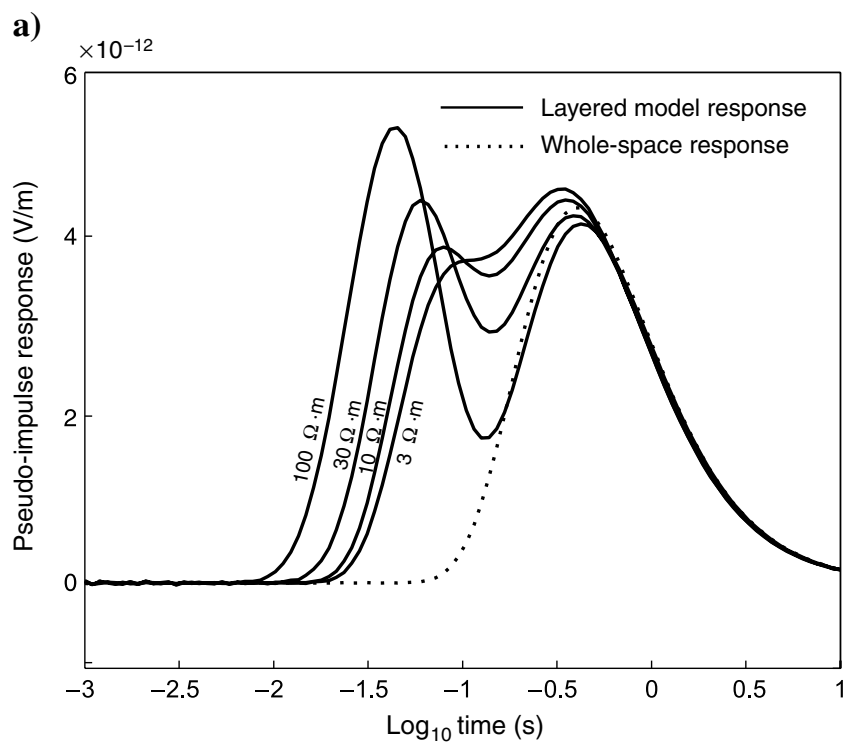

and Knizhermann, 1994), whereas the response of model C was calculated pseudoanalytically by the resistive sheet software described in Swidinsky and Edwards (2009, 2010).

Figure 6a shows the true pseudoimpulse response of model A for a variety of layer resistivities. Figure $6 \mathrm{a}$ also shows a dotted line corresponding to the response of a $0.33 \Omega \cdot \mathrm{m}$ whole-space. Note that the first arrival and the general shape of the early time response is strongly sensitive to the seafloor resistivity, whereas the decay of the late time response is identical for all seafloor resistivites, and thus independent of this particular seafloor model. This is the case even for the wholespace model. Figure $6 \mathrm{~b}$ shows the normalized pseudoimpulse response of model $\mathrm{B}$, along with the responses of uniform half-spaces having resistivities only of the left and right quarter-spaces. Note that all three responses are significantly different, indicating clearly that we are considering a 2D effect from the contact. Note also that the early time information varies

b)

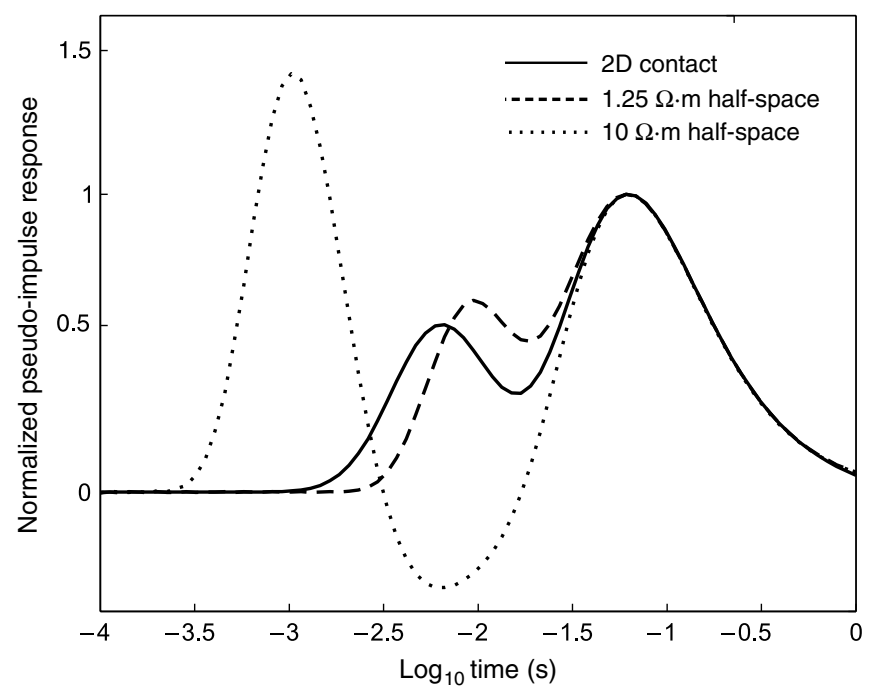

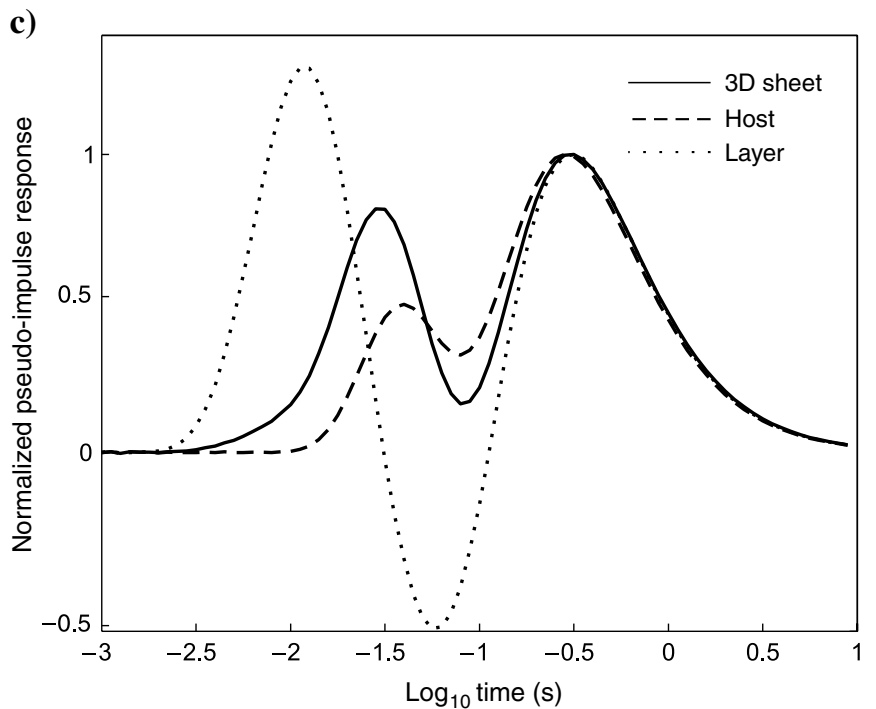

Figure 6. The pseudoimpulse electric field response of models $\mathrm{A}, \mathrm{B}$, and $\mathrm{C}$, respectively. (a) Variable layer resistivity with values of $3 \Omega \cdot \mathrm{m}$, $1 \Omega \cdot \mathrm{m}, 30 \Omega \cdot \mathrm{m}$, and $100 \Omega \cdot \mathrm{m}$. The dotted line shows the response of a $0.33 \Omega \cdot \mathrm{m}$ whole-space. (b) Contact quarter-space response and the two half-space end-member responses for each quarter-space resistivity. (c) Resistive sheet response, host response, and layer response, where the layer has the same resistivity, thickness, and depth as the sheet. 
significantly for the three cases, but that the late time information is identical. We can thus conclude that the late time response is independent of the seafloor resistivity in the presence of this $2 \mathrm{D}$ structure. Finally, Figure $6 \mathrm{c}$ shows the response of model C, the response of only the uniform seafloor host and the response of a layer with the same resistivity, thickness and depth as the sheet. Note that all three responses are significantly different, indicating clearly that we are considering a 3D effect from the sheet. It should be clear from these curves that the early time response is a function of the seafloor resistivity, whereas the late time information is entirely independent of this particular $3 \mathrm{D}$ structure.

\section{RECOVERY OF NAVIGATION DATA}

The intent of the two previous sections was to examine if late time transient marine CSEM behavior is independent of seafloor resistivity structure. Let us now investigate a more practical, real problem: What can this late time response tell us about the position of a receiver cable on the seafloor? Consider again the model shown in Figure 5c; however, imagine that there are now five receivers towed on an $x$-directed cable. The interreceiver spacings are $100,200,400$, and $800 \mathrm{~m}$, and the first receiver is located at $x=600 \mathrm{~m}, y=600 \mathrm{~m}$ (same as the receiver shown in Figure $5 \mathrm{c}$ ). Figure 7 shows the normalized pseudoimpulse response of this fivereceiver cable for the 3D sheet model (solid line), as well as for the host half-space model (dotted line). It is obvious that, at late times, the response is independent of the seafloor resistivity structure for all receivers. Let us now assume that the position of the cable on the seafloor is unknown, but the interreceiver spacing, cable heading, and transmitter position and orientation are well-established. In this case, our navigation problem reduces to two unknown parameters, the $x$ - and $y$-position (or more realistically, the easting and northing) of the first receiver on the cable. Can these two unknowns be determined from the late time data recorded by the array? That

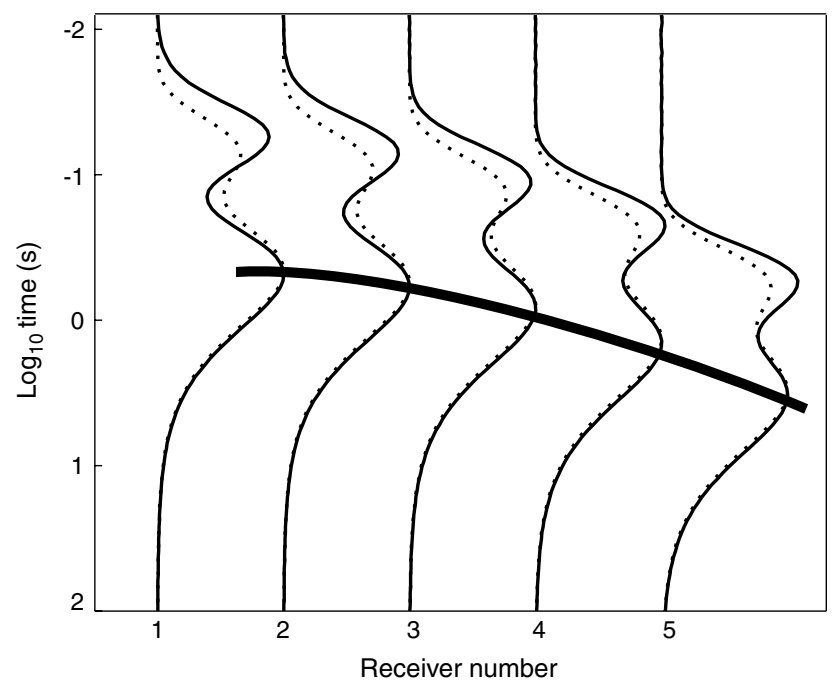

Figure 7. The normalized pseudoimpulse response of a fivereceiver cable for model $\mathrm{C}$. The receivers are located at the $(x$, $y)$ positions $(600 \mathrm{~m}, 300 \mathrm{~m}),(700 \mathrm{~m}, 300 \mathrm{~m}),(900 \mathrm{~m}, 300 \mathrm{~m})$, $(1300 \mathrm{~m}, 300 \mathrm{~m})$, and $(2100 \mathrm{~m}, 300 \mathrm{~m})$. The solid lines correspond to the response of the $3 \mathrm{D}$ model, whereas the dotted line corresponds to the response of the uniform host. The heavy black line shows our subjective distinction between early and late time. is, can we find the cable directly using the electric fields? Following Swidinsky and Edwards (2011), we use eigenparameter statistical analysis to answer this question; however, we use only the data recorded after the times indicated by the heavy black line shown on Figure 7. Because there are two unknown parameters, there must also be two eigenparameters. Transforming $x$ and $y$ into their logarithms (as is common practice), and assuming a 5\% data error on the step response before differentiation, we find that the two eigenparameters and their corresponding percentage errors are given as

$$
\text { Eigenparameter } 1=0.985 \times \log (x)+0.170 \times \log (y)
$$

and

$$
\text { Eigenparameter } 2=0.170 \times \log (x)-0.985 \times \log (y),
$$

with respective percent errors of $0.10 \%$ and $2.44 \%$. This analysis shows that the first eigenparameter is mainly composed of the $x$-position of the cable and that it has an error of less than $1 \%$. The second eigenparameter is mainly composed of the $y$-position of the cable and it has an error of approximately $2.5 \%$. The $x$ - and $y$-coordinates of the cable can thus be determined within acceptable error margins from the late time information recorded by the five receivers. The reader is referred to Swidinsky and Edwards (2011) for a more thorough analysis of such errors. The typical accuracy of an acoustic positioning system is on the order of 1-10 m, and the absolute errors for our example here also fall within this range ( $\pm 1 \mathrm{~m}$ for $x, \pm 7.5 \mathrm{~m}$ for $y$ ). As a general rule of thumb for $n$ receivers, the navigation error will scale as $1 / \sqrt{n}$ in the presence of zero-mean Gaussian noise on the electromagnetic field measurements. Note that these are minimum error estimates, because we have assumed that the seawater conductivity structure is correctly known and there are no timing uncertainties between the transmitter and receivers. However, the former can be determined very well by conductivity-temperature-depth measurements, whereas the latter can be avoided by GPS clock synchronization between the receiver cable and the transmitter.

\section{CONCLUSIONS}

From the previous numerical examples, we have seen that our theoretical model compares well to observations - the late time transient response on the seafloor is governed by induction through the seawater. Let us then conclude by pointing out that the data analysis for the type of configuration shown in Figure 1 is a very straightforward two-step procedure. First, the late time data is inverted to establish the position of the array; the full transient is then used, together with the navigation information, to invert for the seafloor resistivity. This can be done regardless even in the presence of $3 \mathrm{D}$ structures in the seafloor. It should be noted that, because the entire transient shifts in time corresponding to the transmitter-receiver offset, the early time information also contains information on array position. This means that there exists an alternative, even simpler procedure: The entire transient can be inverted simultaneously in one step, with the seafloor resistivity structure and the array position considered as unknowns. Because the late time information is independent of the seafloor resistivity, this procedure will have the same results as the two-step process. The overall conclusions of this study should now be evident: Given a stationary transmitter of well-known position and orientation, and a receiver cable towed on a known 
heading, the position of this cable can be established using the late time transient electric field, even in the presence of 3D seafloor structure.

\section{ACKNOWLEDGMENTS}

The authors would like to thank three anonymous reviewers for their helpful and encouraging comments. Andrei Swidinsky thanks Marion Jegen for financial support.

\section{REFERENCES}

Davydycheva, S., N. Rykhlinski, and P. Legeydo, 2006, Electrical-prospecting method for hydrocarbon search using the induced-polarization effect: Geophysics, 71, no. 4, G179-G189, doi: 10.1190/1.2217367.

Druskin, V. L., and L. A. Knizhnerman, 1994, Spectral approach to solving three-dimensional Maxwell's diffusion equations in the time and frequency domains: Radio Science, 29, 937-953, doi: 10.1029/94RS00747.

Edwards, R. N., 1997, On the resource evaluation of marine gas hydrate deposits using a sea floor transient electric dipole-dipole method: Geophysics, 62, 63-74, doi: 10.1190/1.1444146.
Jones, A., 1988, Static shift of magnetotelluric data and its removal in a sedimentary basin environment: Geophysics, 53, 967-978, doi: 10.1190/ 1.1442533.

Key, K., and A. Lockwood, 2010, Determining the orientation of marine CSEM receivers using orthogonal Procrustes rotation analysis: Geophysics, 75, no. 3, F63-F70, doi: 10.1190/1.3378765.

Mir, R., 2011, Design and deployment of a controlled source EM instrument on the NEPTUNE observatory for long-term monitoring of methane hydrate deposits: Ph.D. thesis, University of Toronto.

Swidinsky, A., and R. N. Edwards, 2009, The transient electromagnetic response of a resistive sheet: Straightforward but not trivial: Geophysical Journal International, 179, 1488-1498, doi: 10.1111/gji.2009.179 issue-3.

Swidinsky, A., and R. N. Edwards, 2010, The transient electromagnetic response of a resistive sheet: An extension to three-dimensions: Geophysical Journal International, 182, 663-674, doi: 10.1111/(ISSN) 1365-246X.

Swidinsky, A., and R. N. Edwards, 2011, Joint inversion of navigation and resistivity structure using a fixed transmitter and a moving, linear receiver array: A preliminary study: Geophysical Journal International, 186, 987-996, doi: 10.1111/j.1365-246X.2011.05104.x.

Weitemeyer, K., 2008, Marine electromagnetic methods for gas hydrate characterization: Ph.D. thesis, University of California, San Diego. 